\title{
TECNOLOGIA DA INFORMAÇÃO NO APOIO À SEGUNDA OPINIÃO FORMATIVA EM SAÚDE PÚBLICA
}

\author{
Monique Lustosa*, Gisele Raptopoulos*, Cibelle Quaglio**, Renato Ribeiro Ferraz**, Lara Jansiski \\ Motta**** \\ Autor correspondente: Cibelle Quaglio. E-mail: cibellequaglio@globo.com \\ * Aluna do Programa de Mestrado em Gestão de Sistemas de Saúde da Universidade Nove de Julho \\ ** Especialista em Odontopediatra pela APCD \\ *** Professor Doutor do Programa de Mestrado em Gestão de Sistemas de Saúde da Universidade Nove de Julho \\ **** Professora Doutora do Programa de Mestrado em Gestão de Sistemas de Saúde da Universidade Nove de Julho
}

\section{Resumo}

O uso da tecnologia da informação em saúde colaborou no avanço das práticas de saúde. A segunda opinião formativa compreende as ações que abrangem um profissional especialista à distância com a utilização de recursos de informática ou telecomunicações para outro profissional. O especialista transmite informações e conhecimento para que o solicitante seja orientado na tomada de decisão clínica. O objetivo deste artigo é analisar a produção científica sobre "segunda opinião formativa em saúde" na literatura brasileira, seguindo uma análise bibliométrica para responder à questão: Como são apresentados os estudos sobre a tecnologia da informação no apoio à segunda opinião formativa em saúde pública? Foram analisados 61 artigos de 2007 a 2014. Foi possível observar que o número de publicações ao longo do período analisado foi crescente. Recomenda-se que, futuros estudos aprofundem a análise e discussão da qualidade e fornecimento das informações prestadas na segunda opinião, uma vez que esta contribui para a descentralização da assistência e maior acesso à saúde em lugares mais distantes.

Palavras-chave: Saúde; Telemedicina; Serviços de saúde; Assistência à saúde. 


\title{
INFORMATION TECHNOLOGY IN SUPPORT OF THE FORMATIVE SECOND OPINION IN PUBLIC HEALTH
}

\begin{abstract}
The use of health information technology helped the advance in health practices. The second formative opinion understands the actions covering a professional expert remotely with the use of computer resources and telecommunications to another professional. The expert transmits information and knowledge so the applicant is directed to the clinical decision making. The objective of this paper is to analyze the scientific production on "second opinion formation in health" in Brazilian literature, following a bibliometric analysis to answer the question: Studies on information technology in supporting the second formative opinion on public health and are being presented? Were analyzed 61 articles from 2007 to 2014 . It can be observed that the number of publications over the period analyzed was growing. It is recommended that future studies deepen the analysis and discussion of the quality and supply of the information provided in the second opinion, since this contributes to the decentralization of care and greater access to health further afield.
\end{abstract}

Keywords: Health; Telemedicine; Health services; Health care.

\section{INTRODUÇÃO}

Nos últimos anos a área da saúde apresentou grandes mudanças e avanços na prevenção, diagnóstico e tratamento das doenças. O uso da tecnologia da informação colaborou para este processo de transformação e inovação, tornando-se realidade a aplicação nos serviços de saúde.(1)

A influência da internet e da telecomunicação proporciona uma importante difusão do conhecimento, podendo ultrapassar barreiras da prática profissional na assistência à saúde. Considerando que a saúde é direito de todos e o Sistema Único de Saúde vigente no Brasil é guiado pela integralidade, equidade e universalidade, o desafio de levar saúde a toda população brasileira pode contar com o papel fundamental desenvolvido pelas inovações tecnológicas, tanto no cuidado com o paciente, na interpretação de exames diagnósticos, sistemas de prevenção às doenças, até mesmo quando a distância territorial apresentar-se como um fator limitante. ${ }^{(2)}$
A telemedicina ou telessaúde é muito utilizada nos países desenvolvidos e sua aplicação apresentou redução de gastos em assistência à saúde em até $60 \%$, colabora com a descentralização dos serviços, diminui a necessidade de gastos com deslocamento de pacientes e de profissionais especializados. (3) O termo refere-se ao uso de tecnologia da informação e comunicação para prover serviços de saúde à distancia. ${ }^{(2)}$ A Organização Mundial de Saúde, em 2011, definiu telemedicina como: a oferta dos serviços de saúde por profissionais lançando mão das tecnologias de informação e comunicação em situações onde a distância é um fator crítico, onde, por meio destas, são realizadas, interações de informações essenciais para diagnóstico, tratamento, prevenção de doenças, além de pesquisas e avaliações como ferramenta de educação continuada dos profissionais que prestam os serviços. ${ }^{(1)}$

As atividades da telemedicina podem ser orientadas em três grupos: (1) teleeducação Interativa e Rede de Aprendizagem Colaborativa; (2) Teleas- 
sistência, Regulação e Vigilância Epidemiológica; e (3) Pesquisa Multicêntrica/Colaboração de Centros de Excelência e da Rede de Teleciência. A primeira designa o uso das tecnologias para ampliação das possibilidades de construção do conhecimento e facilitação do acesso ao material educativo. A teleassistência e Regulação e Vigilância epidemiológica está relacionada à atividade assistencial a distância, integrando educação, vigilância, gestão, processos em saúde. A integração dos centros de pesquisa para compartilhamento dos dados e padronização de métodos é denominada Pesquisa Multicêntrica/Colaboração de Centros de Excelência e da Rede de Teleciência. ${ }^{(4)}$

O Ministério da Saúde identificou, após 2006, relevantes experiências no Brasil a partir da telemedicina e telessaúde. A análise destas experiências culminou no desenvolvimento de um projeto de telessaúde que objetiva a promoção da qualificação dos serviços da Estratégia de Saúde da Família, colaborando para a resolubilidade da atenção básica à saúde. O Programa Rede Telessaúde Brasil foi constituído em 2007 pela Portaria 35 do Ministério da Saúde. A rede é constituída atualmente por 12 núcleos, inseridos em universidades públicas, conectados entre si e interligados a um conjunto de 1171 unidades básicas de saúde. Estas unidades estão distribuídas em regiões distantes de difícil acesso e também nas áreas metropolitanas. O núcleo universitário deve estar vinculado aos cursos de graduação da área da saúde e são responsáveis por teleconsultorias e formulação de segundas opiniões formativas. ${ }^{(5)}$

A teleconsultoria é definida pelo diálogo para discussão de casos clínicos, diagnóstico e procedimentos entre o profissional das Unidades Básicas de Saúde (UBS) e profissionais especialistas. Durante a teleconsultoria o profissional da UBS recebe orientações quanto ao diagnóstico, terapia, laudo, condutas gerais ou propedêuticas do caso em discussão, podendo apresentar dois tipos: teleconsultoria síncrona ou assíncrona. Na teleconsultoria assíncrona as dúvidas são esclarecidas por mensagens off-line, são discutidos os casos eletivos e não complexos, por meio de mensagens eletrôni- cas. Utilizam-se apenas textos com relato do caso pelo médico solicitante e o parecer do médico especialista. ${ }^{(6)}$

$\mathrm{Na}$ teleconsultoria síncrona a discussão é realizada on-line, em tempo real e são discutidos os casos clínicos mais complexos. Neste tipo de teleconsultoria são compartilhados som, imagens e dados do caso clínico, seguido da emissão de laudo com parecer do especialista. ${ }^{(6)}$ A segunda opinião formativa difere da teleconsultoria, é composta de perguntas e respostas normalmente, não obrigatoriamente, resultante de uma teleconsultoria, após passar por avaliação por pares de especialistas, tendo a resposta baseada em levantamento bibliográfico por estes, considerando as melhores evidências científicas e clínicas disponíveis na literatura. ${ }^{(5)}$

Wen ${ }^{(7)}$ define segunda opinião formativa como ações abrangendo um profissional especialista a distância com a utilização de recursos de informática ou telecomunicações para um outro profissional. A formulação da segunda opinião formativa está baseada na problemática real, de forma que possa atender às necessidades específicas dos profissionais das diferentes regiões mais remotas. $O$ profissional especialista transmite informações e conhecimento para que o profissional solicitante seja orientado para o raciocínio e tomada de decisão clínica.

A Portaria $n^{\circ} 402$, de 24 de fevereiro de 2010, considera como Segunda Opinião Formativa a resposta estruturada e sistematizada às perguntas formuladas pelas Equipes de Saúde da Família, a partir dos Pontos Avançados do Telessaúde, sobre temas relacionados ao diagnóstico, planejamento e execução de ações, individuais e coletiva, sobre o processo de trabalho, ou vinculados a casos clínicos atendidos nas Unidades de Saúde da Família. ${ }^{\left({ }^{(8)}\right.}$

De acordo com Haddad(5) para a elaboração das respostas que dão origem a Segunda Opinião formativa, são seguidos os seguintes passos:

1. Seleção das Perguntas pelos especialistas dúvidas clínicas ou no processo de trabalho realizados pelos profissionais da atenção básica. São 
selecionadas as perguntas com base na frequência, relevância e pertinência para a atenção primária e Estratégia de saúde da Família.

2. Produção da resposta Estruturada pelos especialistas-Além de serem respondidas com base em um levantamento bibliográfico e seleção das melhores evidências científicas e clínicas, a resposta é classificada quanto ao grau de evidência científica ( $A, B, C, D)$. Tanto na pergunta, como na resposta, os dados do paciente, pessoa ou grupo envolvidos são mantidos em sigilo.

3. Envio das perguntas e respostas para publicação na Rede Telessaúde Brasil, acompanhada com as referências bibliográficas que a embasaram, a indicação da categoria do profissional que fez a pergunta (médico, enfermeiro, dentista ou outro), a área temática do problema de saúde relacionado à pergunta, o nome do responsável pela resposta $\mathrm{e}$ a data da publicação na rede.

\section{DESCRIÇÃO DA PLATAFORMA}

A Figura 1 ilustra a página inicial para o acesso à Segunda Opinião Formativa da Rede Telessaúde
Brasil. O acesso é feito pelo Portal Biblioteca Virtual em Saúde (BVS) Atenção Primária em Saúde (APS) e Telessaúde Brasil, alocado no servidor da Biblioteca Regional de Medicina (Bireme - Centro Latino-Americano e do Caribe de Informação em Ciências da Saúde da Organização Pan-Americana da Saúde)' com acesso online aberto e com atualização diária. (9) A atualização constante demonstra o potencial de ajuste contínuo das informações com evidências científicas, refletindo as inovações para a área. O Portal BVS APS e Telessaúde Brasil possui uma interface de pesquisa e navegação que compreende conteúdos científicos de forma integrada. O público que acessa o Portal é caracterizado por profissionais envolvidos em processos de tomada de decisão clínica, prestação de serviço de saúde, atenção primária. Adota o modelo de gestão de informação da BVS operando nas seguintes dimensões: Rede Social, Rede de Conteúdo e Rede de Ambientes Aprendizes. A rede social é formada pelas instituições de ensino, membros da Rede Telessaúde e parceiros associados; a rede de conteúdo compreende a coleção de fontes e serviços de informação, e, a rede de ambientes aprendizes permeia as duas primeiras. ${ }^{(5)}$

1 http://www.telessaudebrasil.org.br

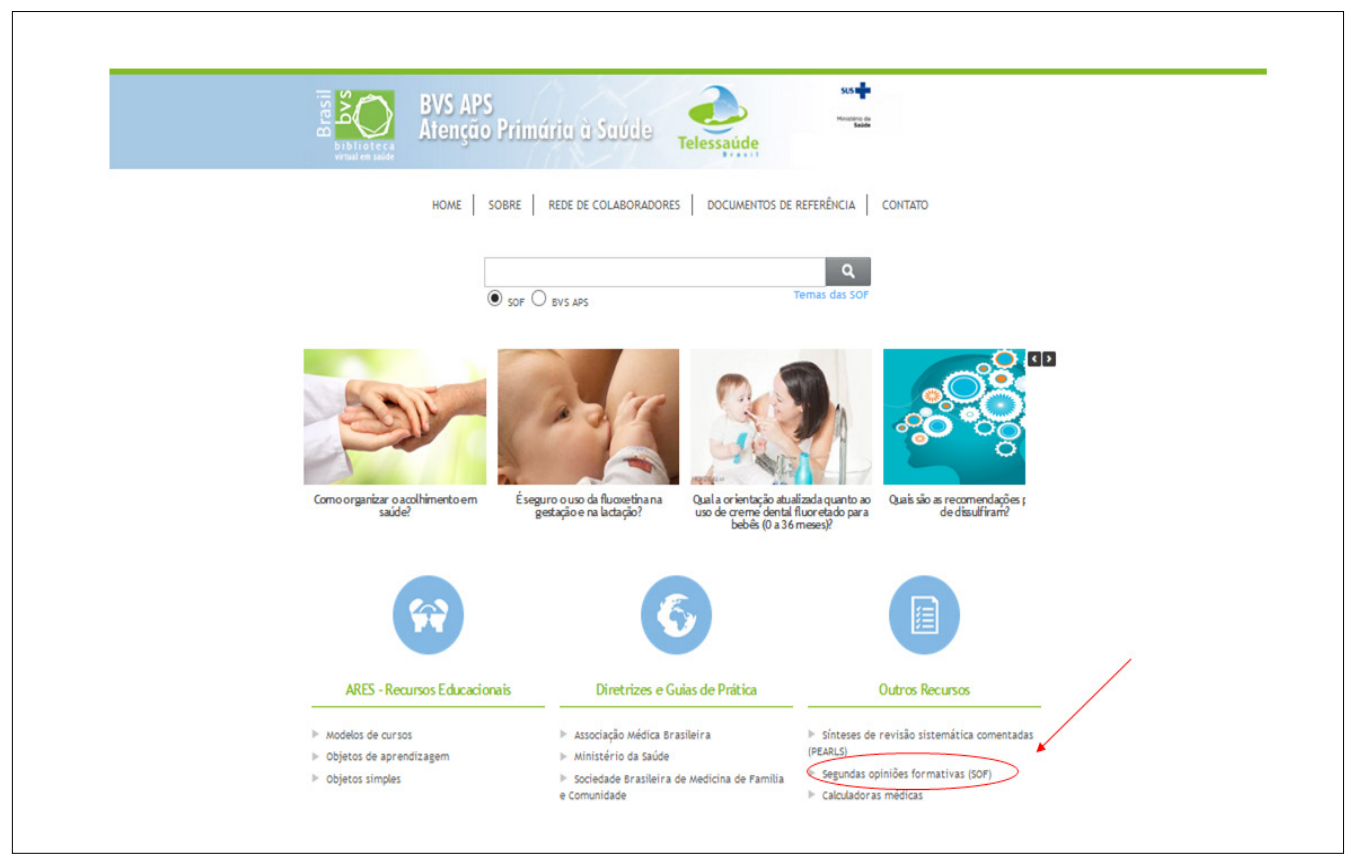

Figura 1 - Ilustração da página inicial de acesso ao Portal BVS APS e Telessaúde Brasil Fonte: Biblioteca Virtual de Saúde. 
Após o acesso inicial, o profissional tem algumas possibilidades para acessar à Segunda Opinião Formativa. Ao clicar no ícone "Segunda Opinião Formativa", sinalizado em vermelho na Figura 1 , serão apresentadas as perguntas e respostas mais frequentes e atuais, conforme Figura 2. O próximo passo é acessar o conteúdo da segunda opinião que compreende a resposta estruturada (diagnóstico e conduta clínica) pelos especialistas, o grau de evidência científica e a bibliografia consultada (Figura 3).

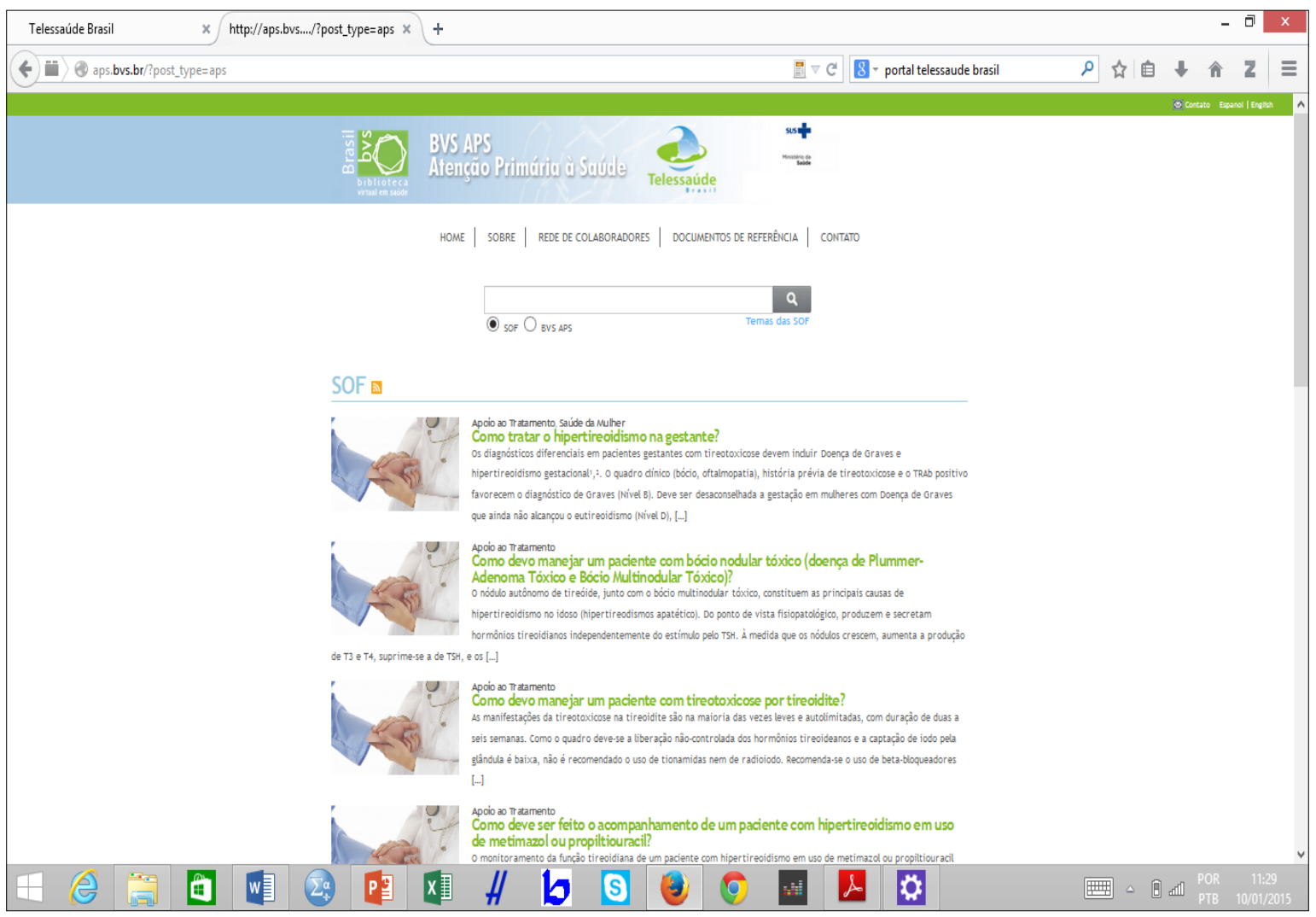

Figura 2 - Página de acesso às perguntas e respostas mais frequentes e atuais de segunda opinião formativa do Portal BVS APS e Telessaúde Brasil

Fonte: Biblioteca Virtual de Saúde.

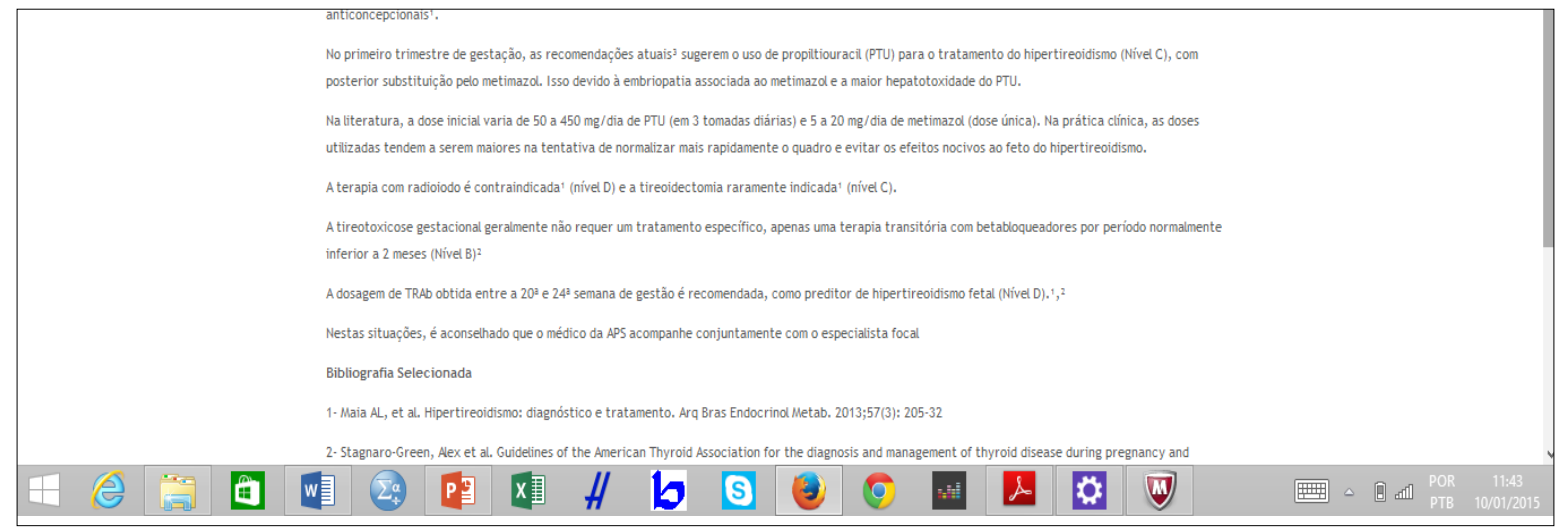

Figura 3 - Resposta estruturada de segunda opinião formativa do Portal BVS APS e Telessaúde Brasil

Fonte: Biblioteca Virtual de Saúde. 
Outras duas maneiras de acessar as respostas de segunda opinião formativa é escolher a área temática de interesse ou digitar o termo relacionado ao problema de saúde na caixa de busca (Figura 4).
A Figura 5 ilustra o exemplo da escolha da temática Saúde Bucal e a apresentação das respostas estruturadas para auxílio do profissional que atua em áreas remotas.

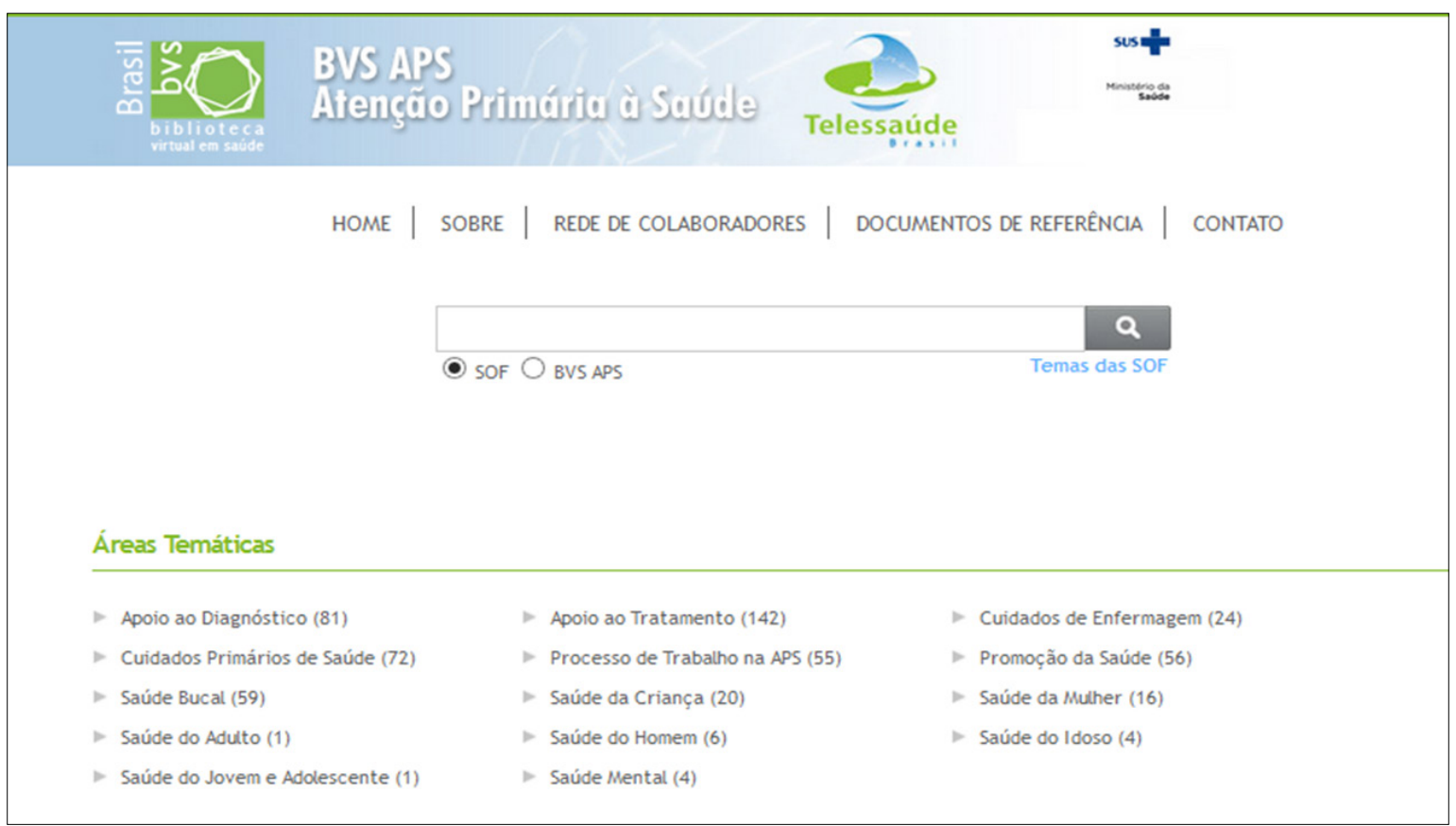

Figura 4 - Tela para acesso às áreas temáticas com o conteúdo da segunda opinião formativa

Fonte: Biblioteca Virtual de Saúde.

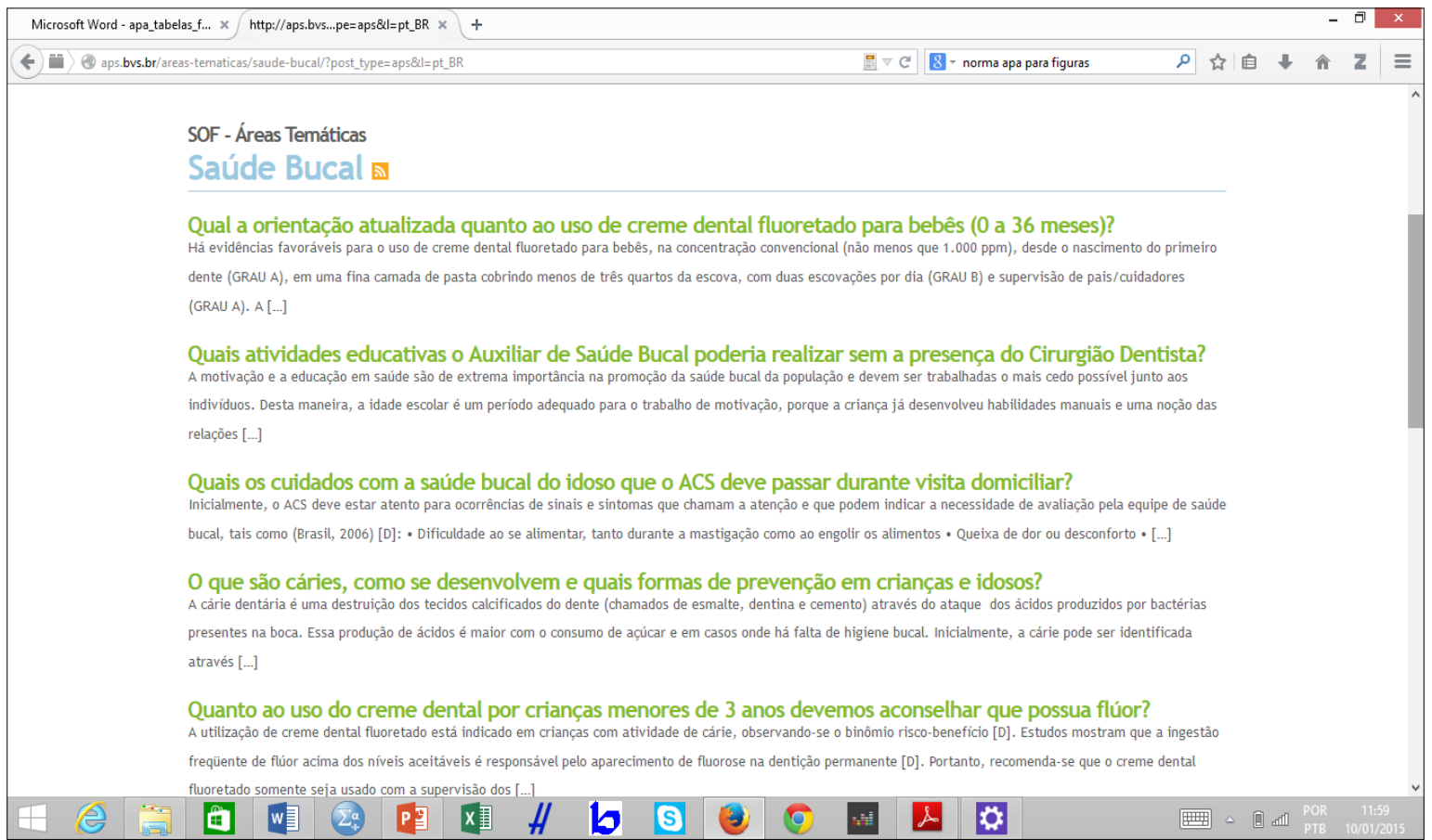

Figura 5 - Tela para acesso às segundas opiniões formativas da área temática de Saúde Bucal

Fonte: Biblioteca Virtual de Saúde. 
A utilização desta ferramenta vem apresentando um crescimento considerável, devido ao fácil acesso à segunda opinião formativa, à possibilidade de interação entre os profissionais da atenção básica e o núcleo de especialistas do Telessaúde Brasil. Segundo Haddad(5) e Abdala(9) em 2010 foram registradas mais de 185 mil visitas e acesso a mais de 976 mil páginas, representando um crescimento de $20 \%$ com relação ao ano anterior. Não foram encontradas publicações com dados mais recentes sobre a visita ao portal e a estes serviços, acredita-se que o aumento do acesso à internet e implantação do Plano Nacional de Banda Larga (PNBL) do Governo Federal, o acesso a estas ferramentas tende a ter aumentado significativamente em relação à 2010.

Considerando que as atividades de Telessaúde apresentam a possibilidade da intervenção com orientação remota especializada no atendimento a um paciente por meio de segunda opinião formativa, torna-se um objeto relevante e importante de investigação científica. Os resultados das experiências e as comprovações da eficácia e efetividade da segunda opinião formativa para melhorar a saúde pública, precisam ser analisados e compartilhados com a comunidade científica, divulgando o acesso destas informações aos profissionais de saúde. O objetivo deste artigo é descrever e analisar a produção científica sobre o assunto "segunda opinião formativa em saúde" na literatura brasileira, seguindo análise bibliométrica para responder à questão: Como está sendo apresentado o desenvolvimento dos estudos sobre a tecnologia da informação no apoio à segunda opinião formativa em saúde pública?"

\section{MÉTODO}

Esta pesquisa é caracterizada como bibliométrica com caráter exploratório e descritivo. Este método permite encontrar os periódicos essenciais que se julgam possuir os trabalhos mais relevantes de um determinado tema, possibilita coletar, selecionar e analisar criticamente os artigos publicados.
Os aspectos metodológicos deste estudo compreenderam três etapas: coleta dos dados, análise e síntese, e, descrição da análise dos dados. Para o primeiro passo foram escolhidas as bases de busca dos artigos e selecionadas as palavras-chave para o levantamento bibliográfico. Como base de busca optou-se pelo Google Scholar, Scielo, BIREME (Biblioteca Regional de Medicina) e BVS. Estas bases foram consideradas por se tratar de uma análise da literatura científica brasileira em periódicos que abrangem o tema relacionado à telemedicina e segunda opinião formativa em saúde. Para a análise da busca no Google Scholar utilizou-se o software Publish or Perish.

Após a identificação das bases de dados, foram estabelecidos os critérios para as buscas. Inicialmente buscou-se pelo termo "telemedicina" e em seguida acrescentado o termo "segunda opinião formativa", delimitando a busca com a utilização do operador booleano AND ("telemedicina" AND "segunda opinião formativa"). O período inicial de busca foi determinado pela data da criação do Programa Rede Telessaúde Brasil e o final o momento da busca realizada pelos pesquisadores compreendendo, assim, as publicações de 2007 a agosto de 2014.

Seguindo a busca inicial, realizou-se uma análise prévia dos artigos encontrados, com a leitura do título e resumo e identificação do periódico ou veículo de publicação. Foram eliminadas as duplicidades e selecionados, a partir desta leitura prévia, os estudos que contemplavam a temática proposta nesta pesquisa e publicados na íntegra para a análise.

$\mathrm{Na}$ etapa de análise, realizou-se a leitura completa dos artigos selecionados; as informações bibliométricas foram organizadas para exploração e síntese dos seguintes dados: autores, fonte da publicação, ano da publicação, tipo de documento e tema central, e, selecionados, estudos relacionados à saúde pública. A partir desta análise realizou-se a síntese e descrição dos resultados por meio de tabelas, gráficos e figuras apresentados na próxima seção deste artigo. 


\section{RESULTADOS E DISCUSSÃO}

A busca inicial resultou em 125 estudos, que após análise prévia, foram excluídas as duplicidades e a partir da análise inicial foram selecionados 61 trabalhos acadêmicos no período de 7 anos. A Tabela 1 apresenta a descrição dos trabalhos selecionados de acordo com o tipo de publicação. Observa-se que a maioria dos estudos que atenderam os critérios de busca desta pesquisa foram publicações em periódicos $(49,2 \%)$, seguido de publicações em anais de evento (37,7\%). Foram consideradas ainda teses e dissertações publicadas na íntegra e outros documentos como resoluções, portarias e publicações dos órgãos públicos relacionados ao assunto.

Tabela 1 - Distribuição da frequência dos estudos selecionados, de acordo com o tipo de publicação

\begin{tabular}{lcc}
\hline \multicolumn{1}{c}{ TIPO de PUblicação } & FreQUÊNCIA & $\%$ \\
\hline Periódicos & 30 & $49,2 \%$ \\
Anais de eventos & 23 & $37,7 \%$ \\
Teses e dissertações & 5 & $8,2 \%$ \\
Outro documento & 3 & $4,9 \%$ \\
Total & 61 & $100,0 \%$ \\
\hline
\end{tabular}

Gráfico 1 - Evolução das publicações ao longo do período estudado

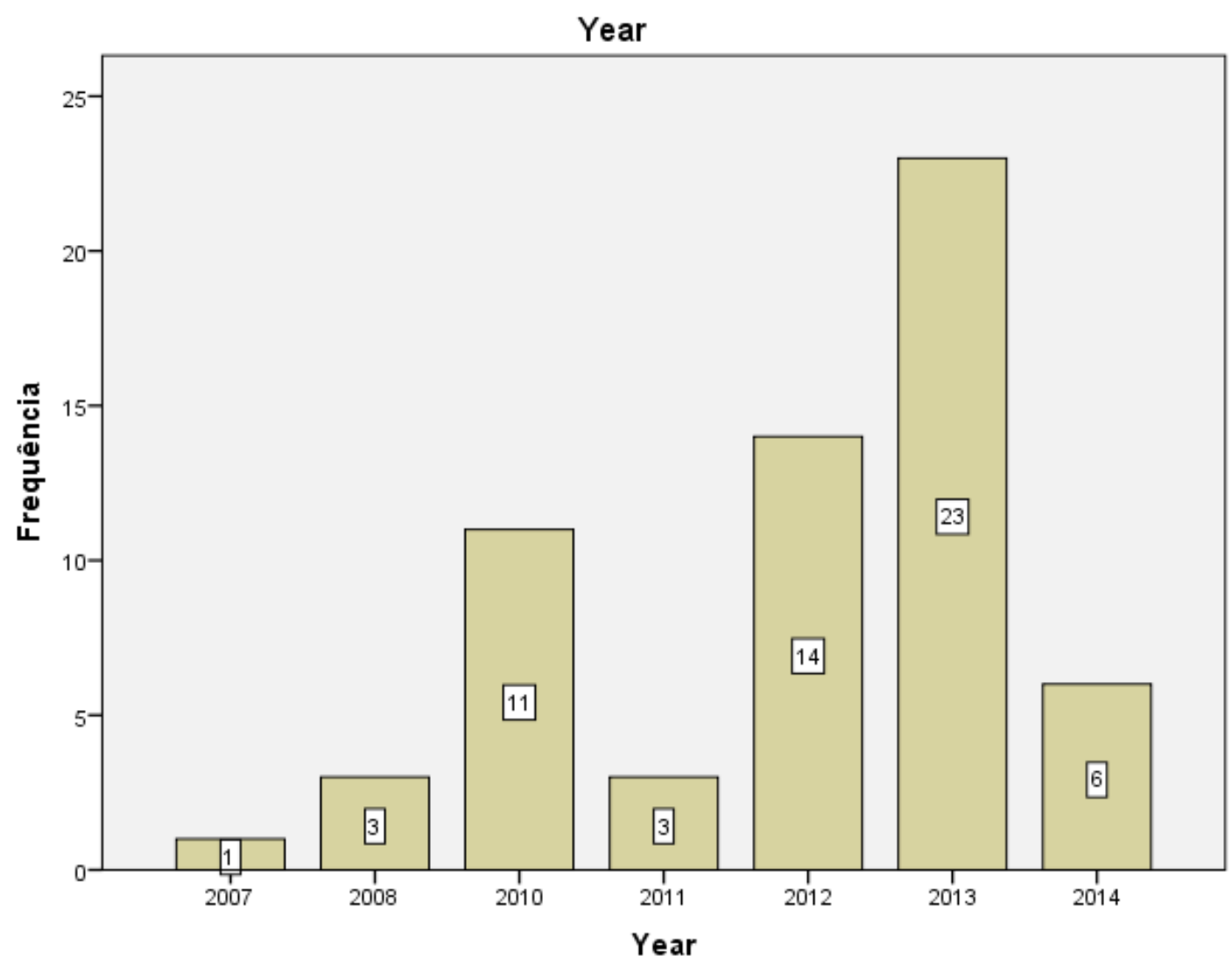


O Gráfico 1 representa a evolução das publicações selecionadas no período determinado nesta pesquisa. É possível observar que nos anos de 2012 e 2013 houve um crescente na produção em relação aos anos anteriores. O que chama a atenção é que no ano de 2011 houve uma queda em relação ao ano anterior de 2010. Em 2014 foram publicados 6 estudos, porém, este ano deve ser considerado incompleto, pois, a data final da pesquisa foi no final do mês de agosto. No ano com o maior número de publicações, 2013, o tipo de publicação mais frequente foram artigos em anais de congresso, $47,8 \%(n=11)$ das publicações deste ano seguido de publicações em periódicos. Estas informações podem ser observadas na Tabela 2 que representa o tipo de publicação por ano.

Tabela 2 - Distribuição dos tipos de estudo ao longo do período analisado

\begin{tabular}{|c|c|c|c|c|c|c|}
\hline \multicolumn{2}{|c|}{ ANO } & ANAIS & DOCUMENTOS & PeRIódicos & Teses & TOTAL \\
\hline 2007 & $\mathrm{~N}(\%)$ & $\mathrm{O}$ & $\mathrm{O}$ & $\mathrm{O}$ & 1 (100\%) & 1 (100\%) \\
\hline 2008 & $N(\%)$ & O & $1(33,3 \%)$ & $2(66,7 \%)$ & $\mathrm{O}$ & $3(100 \%)$ \\
\hline 2010 & $N(\%)$ & $3(27,3 \%)$ & $1(9,1 \%)$ & $7(63,6 \%)$ & $\mathrm{O}$ & 11 (100\%) \\
\hline 2011 & $N(\%)$ & $\mathrm{O}$ & $\mathrm{O}$ & $3(100 \%)$ & $\mathrm{O}$ & 3 (100\%) \\
\hline 2012 & $N(\%)$ & 8 (57,1\%) & $1(7,1 \%)$ & 5 (35,7\%) & 0 & 14 (100\%) \\
\hline 2013 & $N(\%)$ & $11(47,8 \%)$ & $\mathrm{O}$ & $9(39,1 \%)$ & $3(13,0 \%)$ & 23 (100\%) \\
\hline 2014 & $N(\%)$ & $1(16,7 \%)$ & $\mathrm{O}$ & $4(66,7 \%)$ & $1(16,7 \%)$ & $6(100 \%)$ \\
\hline Total & $\mathrm{N}(\%)$ & 23 (37,7\%) & $3(4,9 \%)$ & $30(49,2 \%)$ & $5(8,3 \%)$ & 61 (100\%) \\
\hline
\end{tabular}

Gráfico 2 - Distribuição dos tipos de estudo ao longo do período analisado

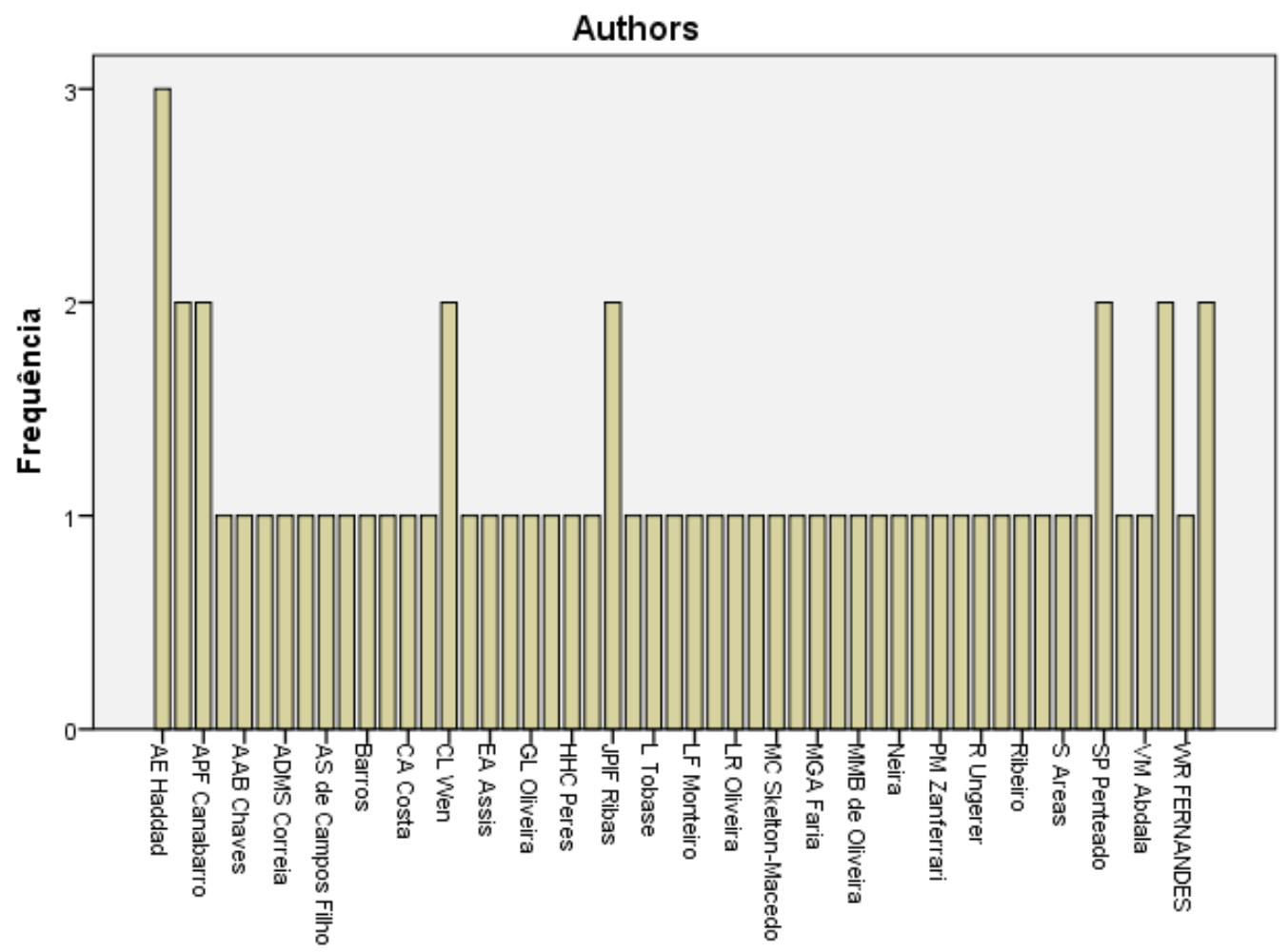

Authors 
Seguindo as análises dos estudos, realizou-se a análise de frequência de autores principais entre as publicações selecionadas. Foram identificados 52 diferentes autores principais na publicação dos 61 trabalhos. O Gráfico 2 ilustra o número de publicações por autor. Nota-se que apenas 8 autores principais apresentaram mais de uma publicação, conforme expressa o Quadro 1. Esta situação provoca uma reflexão, por um lado a grande variedade de autores demonstra que o tema desperta o interesse de vários grupos de estudo no país, porém, espera-se que os estudiosos do tema, apresentem mais de uma publicação, principalmente por se tratar de um período de 7 anos de produção acadêmica. Observa-se também, que apesar de apresentar aumento na produção acadêmica sobre este tema, foram poucas as teses e dissertações publicadas em todo o período. Neste estudo também foram analisados o número de artigos por periódico. As áreas que tiveram o maior número de artigos publicados foram as áreas específicas de telessaúde e telemedicina, porém também foram observados artigos publicados em número significativos na área da enfermagem e saúde pública, conforme observado no Gráfico 3. Este resultado chama atenção para a necessidade de publicações em revistas de outras áreas, tanto da saúde como tecnologia da informação. A enfermagem representa elo importante entre os profissionais médicos e a população, considerada importante área de estudo e divulgação da segunda opinião formativa, porém os resultados poderiam ser apresentados em revistas com abrangência de outras áreas da saúde e as áreas correlatas que apresentam interface com a telemedicina e a segunda opinião formativa.

Quadro 1 - Autores principais com mais de uma publicação sobre o "tema segunda opinião formativa" no período estudado

\begin{tabular}{|c|c|}
\hline Autor PrinciPal & PublicaçõEs \\
\hline Haddad, AE & 3 \\
\hline Spinardi-Panes, AC & 2 \\
\hline Canabarro, APF & 2 \\
\hline Wen, CL & 2 \\
\hline Ribas, JPIF & 2 \\
\hline Penteado, SP & 2 \\
\hline Blasca, WQ & 2 \\
\hline Taveira, ZZ & 2 \\
\hline
\end{tabular}




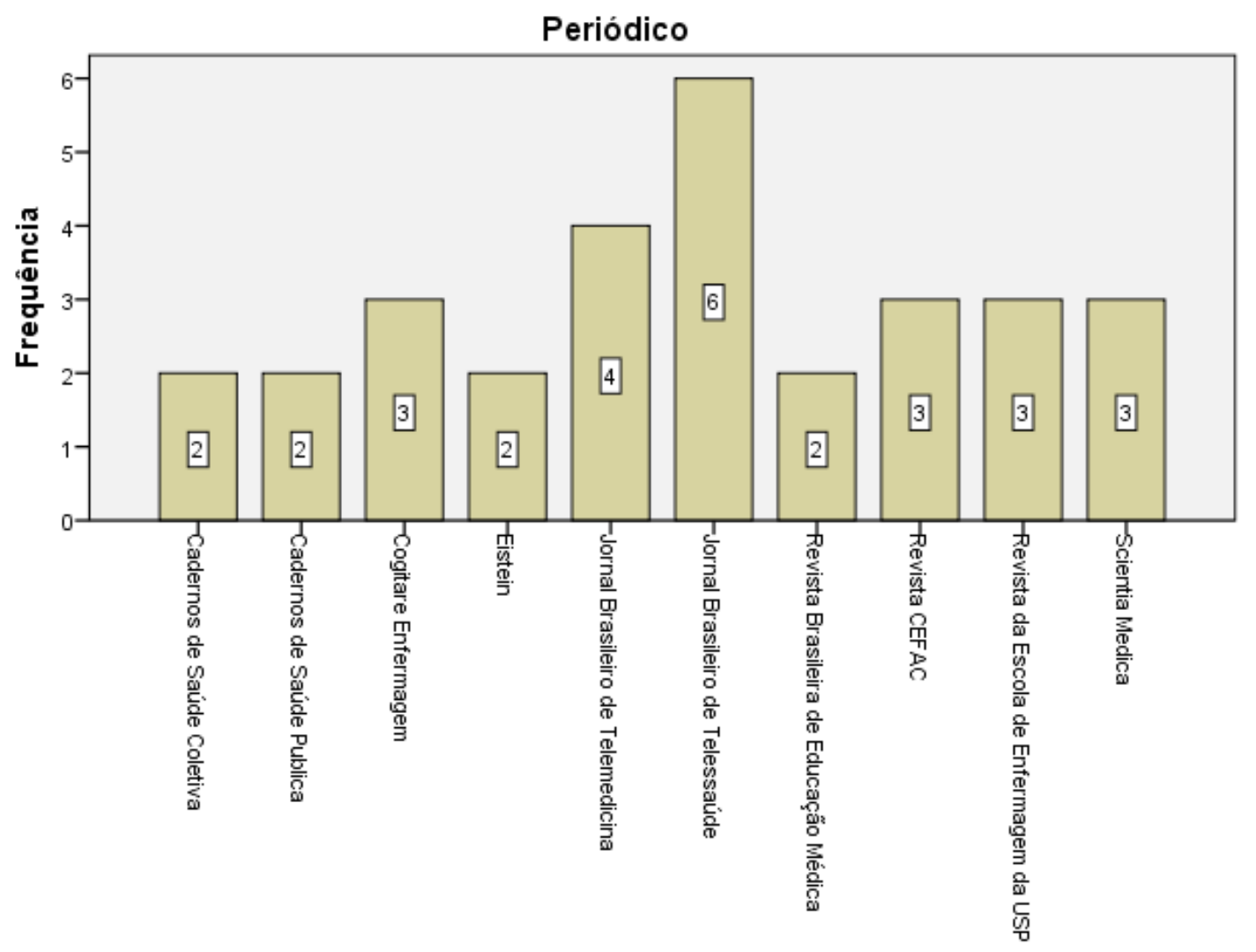

Periódico

Em relação aos temas abordados, após a leitura, são apresentados a seguir, os tópicos mais frequentes nos trabalhos acadêmicos. Um tema muito abordado está relacionado a segunda opinião formativa na cardiologia como descrito em Monteiro et al. ${ }^{(10)}$ Neste trabalho os autores descrevem o desenvolvimento de um aplicativo para sistematizar o modelo de segunda opinião para o diagnóstico através do eletrocardiograma. Por meio deste aplicativo, o profissional pode enviar o traçado do eletrocardiograma juntamente com os dados clínicos do paciente e solicitar a segunda opinião de um especialista ou um grupo de especialistas. Os autores relataram que o aplicativo pode favorecer a assistência às comunidades mais distantes, diminuindo o deslocamento de especialista para a área e o deslocamento do paciente para centros de diagnóstico especializados, resultando na diminuição do ônus à saúde pública e na contribuição para a universalidade da saúde. Cardoso et al.(11) realizaram pesquisa de análise da implantação de siste- ma remoto de segunda opinião em cardiologia em 393 municípios e correlacionaram com os índices de responsabilidade social, observando que estes indicaram maior engajamento dos gestores municipais na implantação do sistema, resultado que demonstra a necessidade de mais estudos envolvendo o desenvolvimento de novas tecnologias para segunda opinião e a gestão dos serviços de saúde.

Ribeiro et al., ${ }^{(12)}$ apresentaram em estudo realizado em Minas Gerais um programa de telecardiologia remoto. As segundas opiniões foram emitidas por uma equipe médica de especialistas e nesta análise os pesquisadores afirmam que os profissionais de saúde adaptaram-se facilmente à ferramenta, foram apresentadas vantagens como possibilidade de diagnóstico precoce e uma redução de $70 \%$ no encaminhamento de pacientes para os centros de referência. Finatto Canabarro et al. ${ }^{(13)}$ apresentaram o foto-telecardiograma como ferramenta de segunda opinião formativa e concluíram 
que a ferramenta com a utilização de smartphone e tablet possibilitaram a formação de segunda opinião formativa e diagnóstico a distância, de maneira confiável.

Além da cardiologia, trabalhos relacionados à dermatologia também foram apresentados de maneira relevante na literatura científica. Silva et al., (14) Trindade et al. ${ }^{(15)}$ e Ribas et al. ${ }^{(4)}$ analisaram a concordância entre o diagnóstico presencial e a segunda opinião remota em doenças de pele por meio de imagens digitais à distância. Em todos os estudos os autores observaram alto grau de concordância variando de $80 \%$ a $92 \%$, sendo considerada ótima concordância pelos pesquisadores.

Em uma revisão sistemática, Padilha et al., (7) ao analisarem os trabalhos incluídos na revisão, sugerem que as ações relacionadas à segunda opinião formativa contribuíram para a descentralização da assistência e maior acesso à saúde em lugares mais distantes.

Outras publicações mostram estudos em outras áreas, como a de fonoaudiologia (16-18) e trabalhos multidisciplinares. $(5,19,20)$

\section{CONSIDERAÇÕES FINAIS}

A utilização da Tecnologia da Informação e Comunicação na área da saúde, demonstra que tem aumentado a capacidade de apoiar a segunda opinião formativa em saúde, colaborando para que os profissionais, mesmo distantes de grandes centros referenciados, possam proporcionar assistência mais abrangente à população, atendendo os princípios do Sistema Único de Saúde (SUS).

Este estudo teve o objetivo de analisar a produção acadêmica brasileira relacionada ao tema e sua aplicação em saúde pública. As publicações apresentaram-se crescente ao longo do período, no entanto, grande parte dos trabalhos destacam o tema apenas no referencial teórico.

Este estudo evidenciou que nas publicações, os assuntos mais prevalentes estão direcionados a determinadas áreas da saúde, como a cardiologia, por exemplo. A análise sugere assuntos que possam emergir para futuros projetos de pesquisas, como estudos relacionados ao custo-efetividade da utilização da segunda opinião formativa, considerando que esta prática possa diminuir significativamente o deslocamento dos pacientes para grandes centros, o diagnóstico precoce pode tornar o cuidado menos custoso e consequentemente promovendo saúde em maior parte do território nacional.

Notou-se que as publicações nesta área, apresentam-se ainda incipientes e ainda precisam ser mais exploradas. Recomenda-se que, os futuros estudos, aprofundem a análise e discussão da qualidade e fornecimento das informações prestadas na segunda opinião, sua aceitação pelos profissionais de saúde e o impacto das ações nos índices e indicadores em saúde pública.

\section{REFERÊNCIAS}

1. Padilha EZ, Silva FRD e Pontes FS. Segunda opinião especializada educacional no telessaúde Brasil: uma revisão. Publicatio UEPG: Ciências Biológicas e da Saúde. 2013;19(1): 3946.

2. Wen CL. Telemedicina e telessaúde- um panorama no Brasil. Informática Pública. 2008;10(2):7-15.

3. Robson RR. Sistema de eletrocardiografia de baixo custo baseada em plataformas móveis para aplicação em Telemedicina [dissertação]. São Luís: Universidade Federal do Estado do Maranhão; 2007.

4. Ribas J, Cunha MDGS, Schettini APM, Ribas CBR. Concordância entre diagnósticos dermatológicos obtidos por consulta presencial e por análise de imagens digitais. An. bras. dermatol. 2010;85(4):441-447.

5. Haddad AE. Experiência brasileira do Programa Nacional Telessaude Brasil. Mathias I, Monteiro A. Gold Book [on-line]: inovação tecnológica em educação e saúde. Rio de Janeiro: EdUERJ; 2012. Disponível em: http://www.telessaude.uerj. br/resource/goldbook/pdf/2.pdf. 
6. Barros K. Avaliação de usabilidade dos sistemas computacionais utilizados nos serviços de teleconsultoria do BHTelessaúde [dissertação]. Belo Horizonte: Universidade Católica de Minas Gerais; 2007.

7. Wen CL. Telemedicina e telessaúde: inovação e sustentabilidade. In: Monteiro A, Neves J, organizadores. Inovação tecnológica em educação em saúde. Rio de Janeiro: UERJ; 2011.

8. BRASIL. Portaria n. $4 \mathrm{O} 2$ de 24 de fevereiro de 201O. Institui, em âmbito nacional, o Programa Telessaúde Brasil para apoio à Estratégia de Saúde da Família no Sistema Único de Saúde, institui o Programa Nacional de Bolsas do Telessaúde Brasil e dá outras providências. Diário Oficial da União, Brasília (DF), 25 fev. 2010, Seção 1,. 36.

9. Abdala CVM. Construindo a inteligência coletiva nas redes e fluxos locais de informação do Programa Telessaúde Brasil. In: Monteiro A, Neves J, organizadores. Inovação Tecnológica em Educação em Saúde. Rio de Janeiro: EdUERJ; 2001.

10. Monteiro LF, Fialla MW, Simões PWTDA, Moretti GP, Casagrande RS, Salvaro RG et al. Informática em saúde - segunda opinião formativa via eletrocardiografia digital e informações clínicas de um Sistema de Registro Eletrônico em Saúde. In: Anais do $5^{\circ}$ Congresso Sul-Brasileiro de Computação; 29 set-Ol out.2010; Criciúma. Criciúma: Universidade do Extremo Sul Catarinense; 2010. Disponível em: http://periodicos.unesc.net/sulcomp/article/ view/265/271

11. Cardoso CS, Ribeiro AL, Castro RL, César CC, Caiaffa WT. Implementation of a cardiology care program in remote areas in Brazil: influence of governability. Rural remote health. 2010;10(3):1472.

12. Ribeiro ALP, Alkmim MB, Cardoso CS, Carvalho GGR, Caiaffa WT, Andrade MV et al.
Implementation of a telecardiology system in the state of Minas Gerais: the Minas Telecardio Project. Arq. bras. cardiol. 2010; 95(1):70-78.

13. Finatto Canabarro AP, Schwalbert ÂM, Bertoglio Cardoso R, Valdez CF, Sturm Trindade C, W de Oliveira H et al. Avaliação do foto-eletrocardiograma como ferramenta de segunda opinião formativa. Sci. med. 2O13;23(4).

14. Silva CS, Souza MB, Duque IA, Medeiros LM, Melo NR, Araújo CA et al. Teledermatologia: correlação diagnóstica em serviço primário. An. bras. dermatol. 2009;84(5):489-93.

15. Trindade MÂB, Wen CL, Neto CF, Escuder MM, Andrade VLG, Yamashitafuji TMT et al. Accuracy of store-and-forward diagnosis in leprosy. J. telemed. telecare. 2008; 14(4): 2O8210.

16. Blasca WQ, Maximino LP, Galdino DG, Campos K e Picolini MM. Novas tecnologias educacionais no ensino da Audiologia. Rev. CEFAC. 2010;12(6):1017-24.

17. Spinardi-Panes AC, Lopes-Herrera SA e Maximino LP. Aspectos éticos e legais na prática da telessaúde em fonoaudiologia. Rev. CEFAC. 2013;15(4):1040-1043.

18. Torres-Pereira C, Possebon RS, Simões A, Bortoluzzi MC, Leão JC, Giovanini AF et al. Email for distance diagnosis of oral diseases: a preliminary study of teledentistry. J. telemed. telecare. 2008;14(8):435-438.

19. Machado FSN, de Carvalho MAP, Mataresi A, Mendonça ET, Cardoso L M, Yogi M $S$ et al. Utilização da telemedicina como estratégia de promoção. Ciênc. saúde coletiva. 2010;15(1):247-254.

2O. Barbosa AK, Novaes M, Stamford P, Queiróz $A E$, Morais G, et al. HealthNet: um sistema integrado de telediagnóstico e segunda opinião médica. News Generation. 2010; 5(4). 